\title{
Abundance and Community Structure of Bacteria on Asian Dust Particles Collected in Beijing, China, during the Asian Dust Season
}

\author{
Nobuyasu Yamaguchi, ${ }^{* a}$ Takashi Baba, ${ }^{a, b}$ Tomoaki Ichijo, ${ }^{a}$ Yuka Himezawa, ${ }^{c}$ Kanami Enoki, ${ }^{c}$ \\ Makoto Saraya ${ }^{a, d}$ Pin-Fang Li, ${ }^{e}$ and Masao $\mathrm{Nasu}^{a}$ \\ ${ }^{a}$ Graduate School of Pharmaceutical Sciences, Osaka University; 1-6 Yamadaoka, Suita, Osaka 565-0871, \\ Japan: ${ }^{b}$ Faculty of Agriculture, Tottori University; 4-101 Koyamacho-minai, Tottori 680-8553, Japan: \\ ${ }^{c}$ School of Pharmaceutical Sciences, Osaka University; 1-6 Yamadaoka, Suita, Osaka 565-0871, Japan: \\ ${ }^{d}$ Faculty of Pharmaceutical Sciences, Himeji Dokkyo University; 7-2-1 Kamiohno, Himeji, Hyogo 670-8524, \\ Japan: and ${ }^{e}$ College of Resources and Environmental Sciences, China Agricultural University; \\ 2 Yuanmingyuan, West Road, Beijing 100193, China. \\ Received July 22, 2015; accepted October 19, 2015
}

\begin{abstract}
Approximately $180 \mathrm{t} / \mathrm{km}^{2}$ of Asian dust particles are estimated to fall annually on Beijing, China, and there is significant concern about the influence of microbes transported by Asian dust events on human health and downwind ecosystems. In this study, we collected Asian dust particles in Beijing, and analyzed the bacterial communities on these particles by culture-independent methods. Bacterial cells on Asian dust particles were visualized first by laser scanning microscopy, which demonstrated that Asian dust particles carry bacterial cells to Beijing. Bacterial abundance, as determined by quantitative polymerase chain reaction (PCR), was $10^{8}$ to $10^{9}$ cells/g, a value about 10 times higher than that in Asian dust source soils. Interseasonal variability of bacterial community structures among Asian dust samples, as compared by terminal restriction fragment length polymorphism (T-RFLP), was low during the Asian dust season. Several viable bacteria, including intestinal bacteria, were found in Asian dust samples by denaturing gradient gel electrophoresis (DGGE). Clone library analysis targeting 16S ribosomal RNA (rRNA) gene sequences demonstrated that bacterial phylogenetic diversity was high in the dust samples, and most of these were environmental bacteria distributed in soil and air. The dominant species in the clone library was Segetibacter aerophilus (Bacteroidetes), which was first isolated from an Asian dust sample collected in Korea. Our results also indicate the possibility of a change in the bacterial community structure during transportation and increases in desiccation-tolerant bacteria such as Firmicutes.
\end{abstract}

Key words Asian dust; bacteria; long-range transportation; laser scanning microscopy; 16S ribosomal RNA (rRNA) gene library; phylogenetic analysis

Aeolian dust primarily occurs in arid and semi-arid regions. When a wind-sand stream occurs, dust particles can be lifted and transported over long distances by air currents. Aeolian dust particles are thought to be carriers of microbes, and abundance and community composition of microbes transported with aeolian dusts have been reported to clarify their possible impacts on public health and ecosystems. ${ }^{1-5)}$ Major aeolian dust events arise from the Sahara and Sahel deserts (African dust), Australian deserts (Australian dust), and the Taklamakan Desert, Gobi Desert and Loess Plateau (Asian dust). It is well known that Asian desert dust particles are transported for long distances, ${ }^{6)}$ even reaching the North American Continent (more than $15000 \mathrm{~km}$ away). ${ }^{78}$ Asian dust particles can sometimes be transported globally in $13 \mathrm{~d}^{9)}$ and have been identified in ice and snow cores of Greenland ${ }^{10)}$ and the French Alps. ${ }^{11)}$

In China, Asian dust has caused disasters as sand storms. ${ }^{12)}$ Approximately $180 \mathrm{t} / \mathrm{km}^{2}$ of Asian dust particles are estimated to fall annually on Beijing, China. ${ }^{13)}$ There are significant concerns regarding the health effects of Asian dust ${ }^{14-18)}$ as well as the influence of microbes transported by Asian dust events on plant, animal and human diseases and on downwind

The sequences obtained in this study have been deposited in the DNA Data Bank of Japan (DDBJ) and assigned accession numbers (LC026811LC026943). ecosystems. ${ }^{19-22)}$ To estimate the risk of bacteria transported by Asian dust events on human health and downwind ecosystems, the abundance and community structure of bacteria carried on Asian dust particles should be accurately determined. It is well-known that most (usually more than $90 \%$ ) bacteria in the natural environment are difficult to culture under conventional conditions ${ }^{23)}$ and thus culture-independent approaches are required for analysis of bacterial dynamics in atmospheric environments. ${ }^{24,25)}$

In this study, we collected Asian dust particles in Beijing, and visualized bacterial cells on Asian dust particles by laser scanning microscopy to directly demonstrate that Asian dust particles carry bacterial cells. We determined bacterial abundance by quantitative polymerase chain reaction (PCR) targeting the eubacterial $16 \mathrm{~S}$ ribosomal RNA (rRNA) gene and compared bacterial community profiles by terminal restriction fragment length polymorphism (T-RFLP) among Asian dust samples to investigate inter-seasonal variability of bacterial community structure during the Asian dust season. We also analyzed bacterial communities before and after culturing with four media to estimate viable bacterial genera and species by denaturing gradient gel electrophoresis (DGGE) based on bacterial 16S rRNA gene sequences. Finally we analyzed bacterial community structure by clone library analysis targeting 16S rRNA gene sequences to determine the dominant bacteria in Asian dust samples collected in Beijing. 


\section{MATERIALS AND METHODS}

Collection of Asian Dust Particles in Beijing Asian dust samples that fell on the top of a building ( $c a .10 \mathrm{~m}$ in height) were collected at the China Agricultural University in Beijing (Figs. 1A, B). Dust particles that fell into a sterilized stainless-

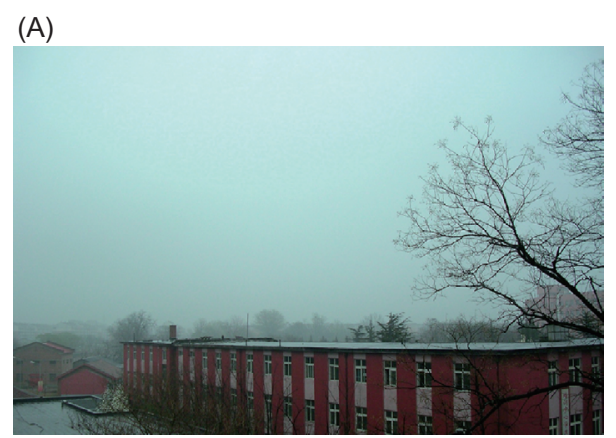

(B)

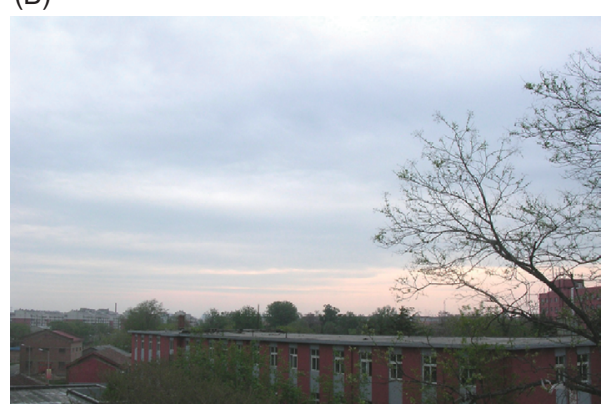

(C)

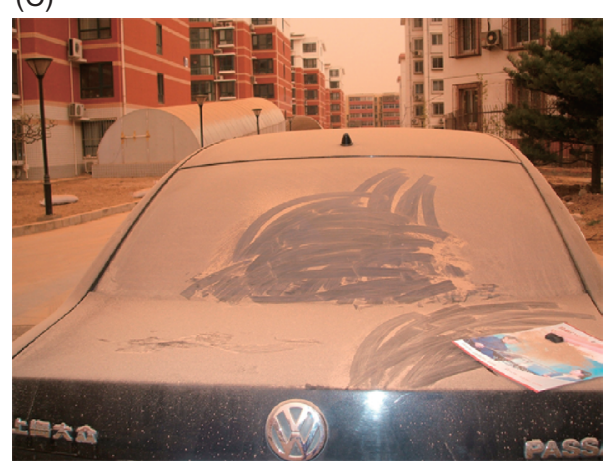

Fig. 1. Observation from the Sampling Point (Roof Top at China Agricultural University, Beijing, China; $c a$. $10 \mathrm{~m}$ in Height) on an Asian Dust Day (A) and a Non-Asian Dust Day (B)

When a severe Asian dust event occurred, a car was covered with dust particles within one night (C). steel bucket over a $24 \mathrm{~h}$ period were collected. Twelve samples were obtained on different days in the Asian dust season between 20 March and 11 May, 2010. When severe Asian dust events occur in Beijing, dust particles could cover a car (Fig. 1C), and more dust particles were collected within $24 \mathrm{~h}$ on 20 March and 10 May than on the other sampling days.

Visualization of Microbial Cells on Asian Dust Particles Ten milligram of the dust samples was suspended in phosphate-buffered saline (PBS) $(\mathrm{pH} 7.2)$ containing 4\% (w/v) paraformaldehyde and fixed at $4^{\circ} \mathrm{C}$ for $16 \mathrm{~h}$. After fixation, dust suspensions were filtered through sterilized $0.4 \mu \mathrm{m}$-pore size polycarbonate membrane filters (K040A025A; Toyo Roshi Kaisha, Tokyo, Japan) and rinsed twice with particle-free water. The filters were then dehydrated in an ethanol series (50, 80 and 100\% ethanol for $3 \mathrm{~min}$ each) and dried under vacuum. The filters were stained for $10 \mathrm{~min}$ with nucleic acidstaining dye, SYBR Green I (Life Technologies, Carlsbad, CA, U.S.A.; 1/10000-dilution of the supplied product) containing $2 \%$ Tween 20 . After washing with particle-free water (3 times, $10 \mathrm{~min}$ each), the filters were mounted in immersion oil for observation by laser scanning microscopy (TCS-SP5; Leica Microsystems, Wetzlar, Germany). The microspectrophotometer of the laser scanning microscope was used to discriminate microbial cells from dust particles. ${ }^{26)}$ All particles were observed under blue excitation (wavelength $480 \mathrm{~nm}$ ) according to the optimized protocol ${ }^{26)}$ and particles with green fluorescence (wavelength 500-540 nm) were detected as microbial cells; those with yellow to red fluorescence (wavelength $550-680 \mathrm{~nm}$ ) were detected as non-biological particles such as soil particles. ${ }^{26)}$

Direct DNA Extraction Total nucleic acids were extracted from $0.5 \mathrm{~g}$ of each Asian dust samples using a FastDNA Spin Kit for soil (MP Biomedicals, Santa Ana, CA, U.S.A.) according to the manufacturer's instructions. The DNA suspension was purified using a Wizard DNA clean-up system (Promega, Fitchburg, WI, U.S.A.) according to the manufacturer's instructions.

Quantitative Real-Time PCR Bacterial abundance was quantified by real-time PCR targeting 16S rRNA genes with a LightCycler (Roche Diagnostics, Basel, Switzerland). Realtime PCR was performed with universal primer sets EUB f933 (5'-GCACAA GCGGTGGAGCATGTGG-3') and EUB r1387 (5'-GCCCGGGAACGT ATT CACCG-3' $)^{27)}$ according to the procedure reported by Nishimura et al. ${ }^{28)}$ To determine the recovery rate of DNA during extraction, known amounts of DNA fragment of the luciferase gene $(l u c)$ were inoculated
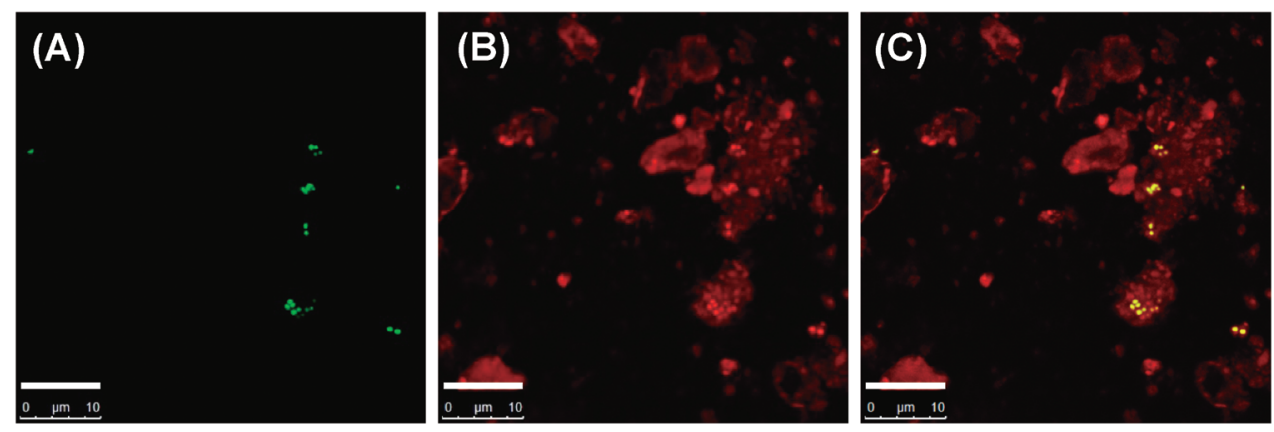

Fig. 2. Discrimination of Bacterial Cells on Asian Dust Particles by Laser Scanning Microscopy Following Fluorescent Nucleic Acid Staining (Scale Bar: $10 \mu \mathrm{m}$ )

(A) Green fluorescence from bacterial cells under blue excitation. (B) Red fluorescence from Asian dust particles under blue excitation. (C) Composite image. 
into the samples before DNA extraction as an internal standard and quantified after DNA extraction. ${ }^{28)}$ The DNA recovery rate was calculated by comparing the copy number of the inoculated $l u c$ gene before and after DNA extraction. The copy number of the $16 \mathrm{~S}$ rRNA gene quantified by real-time PCR was calibrated based on the DNA recovery rate.

T-RFLP Analysis For the T-RFLP analysis, 16S rRNA gene sequences of the bacterial population were amplified by PCR using the universal primers Cy5-labeled-8f and EUB926r. ${ }^{29)}$ PCR for T-RFLP analysis was performed according to the procedure reported by Yamaguchi et al. ${ }^{30)} \mathrm{PCR}$ products were purified and concentrated using MiniElute PCR purification Kit (QIAGEN, Hilden, Germany). The purified PCR products were then digested with MspI (TaKaRa Bio, Shiga, Japan) for $3 \mathrm{~h}$ at $37^{\circ} \mathrm{C}$. DNA was precipitated with ethanol and the DNA pellet was then suspended in CEQ Sample Loading Solution (Beckman Coulter, Brea, CA, U.S.A.). CEQ DNA Size Standard-600 (Beckman Coulter) was added as an internal standard and fluorescence labeled terminal restriction fragments (T-RFs) were size separated by capillary electrophoresis using the CEQ8000 (Beckman Coulter). T-RFs shorter than $90 \mathrm{bp}$ were excluded from the analysis to avoid uncertainties associated with fragment size determination as reported by Hodges and Olson. ${ }^{31)}$ Most peaks derived from dust samples were larger than $90 \mathrm{bp}$. The measured peak profiles were analyzed using the database MiCA3 (http://mica. ibest.uidaho.edu/rundigest.php).

DGGE Analysis To identify active bacterial species, DGGE analysis was performed according to the procedure reported by Iwamoto et al. ${ }^{27)}$ on both directly extracted bacterial DNA and bacterial DNA extracted from cultured Asian dust samples. To culture bacterial cells in Asian dust samples collected on 20 March and 10 May, $10 \mathrm{mg}$ of each dust sample was inoculated into $9 \mathrm{~mL}$ of four media; standard medium $(5 \mathrm{mg} / \mathrm{mL}$ peptone, $2.5 \mathrm{mg} / \mathrm{mL}$ yeast extract, and $1 \mathrm{mg} / \mathrm{mL}$ glucose), $1 \%$ standard medium, $0.05 \%$ yeast extract, and R2A medium (Nihon Pharmaceutical, Osaka, Japan). Samples were incubated at $25^{\circ} \mathrm{C}$ for $7 \mathrm{~d}$ with slow shaking, and then bacterial DNA was extracted and purified using the Wizard Genomic DNA Purification Kit (Promega) according to the manufacturer's instructions. DGGE and sequencing of DGGE fragments were performed according to the procedure reported by Yamaguchi et al. $^{32)}$

Random Cloning The 16S rRNA gene library was constructed from the Asian dust sample collected on 20 March. Nearly full-length 16S rRNA gene sequences of the bacterial domain were amplified by PCR using the universal primers 8f (5'-AGAGTT TGA TCC TGGCTCAG-3') and 1492r (5'-TACCTTGTTACGACT T-3'), according to the procedure reported by Yamaguchi et al. ${ }^{26)} 16 \mathrm{~S}$ rRNA gene fragments of more than 150 randomly selected clones were sequenced at Fasmac (Kanagawa, Japan). No clones were obtained when DNA suspension was not added. Sequences were analyzed with the ribosomal database project ${ }^{33)}$ and clustered as operational taxonomic units (OTUs) based on a 97\% cutoff. Rarefaction curves were constructed with Distance-Based OTU and Richness (DOTUR) at $85 \%$ similarity. ${ }^{34}$

\section{RESULTS AND DISCUSSION}

Visualization of Microbial Cells on Asian Dust Particles
Table 1. Bacterial Abundance in Asian Dust Samples Collected in Beijing, China, Determined by Quantitative PCR Targeting the 16S rRNA Gene

\begin{tabular}{rcc}
\hline \hline Samples & $\begin{array}{r}16 \mathrm{~S} \text { rRNA gene } \\
(\text { copies/g) }\end{array}$ & $\begin{array}{c}\text { Estimated bacterial number* } \\
(\text { cells/g) }\end{array}$ \\
\hline 20 Mar. & $1 \times 10^{9}$ & $2 \times 10^{8}$ \\
21 Mar. & $4 \times 10^{9}$ & $8 \times 10^{8}$ \\
23 Mar. & $1 \times 10^{9}$ & $2 \times 10^{8}$ \\
24 Mar. & $8 \times 10^{8}$ & $2 \times 10^{8}$ \\
27 Mar. & $9 \times 10^{9}$ & $2 \times 10^{9}$ \\
4 Apr. & $4 \times 10^{9}$ & $8 \times 10^{8}$ \\
9 Apr. & $8 \times 10^{9}$ & $2 \times 10^{9}$ \\
10 Apr. & $4 \times 10^{9}$ & $8 \times 10^{8}$ \\
29 Apr. & $1 \times 10^{9}$ & $2 \times 10^{8}$ \\
7 May & $6 \times 10^{8}$ & $1 \times 10^{8}$ \\
10 May & $1 \times 10^{10}$ & $2 \times 10^{9}$ \\
11 May & $4 \times 10^{9}$ & $8 \times 10^{8}$ \\
\hline
\end{tabular}

* 16S rRNA gene copy number: 5 copies/cell.

Microbial cells on Asian dust particles collected in Beijing were visualized by laser scanning microscopy using an optimized protocol ${ }^{26)}$ following fluorescent nucleic acid staining with SYBR Green I (Fig. 2). We confirmed that microbial cells were attached to big dust particles $(>5 \mu \mathrm{m})$ and several microbial cells were attached to one of these 'big' particles (Fig. 2). No microbial cells were attached to small particles $(<5 \mu \mathrm{m})$. These data directly demonstrate that Asian dust particles carry microbial cells to Beijing.

Bacterial Abundance on Asian Dust Particles Collected in Beijing Bacterial abundance on Asian dust particles was determined by quantitative real-time PCR targeting the $16 \mathrm{~S}$ rRNA gene with universal primer sets. Asian dust samples collected in Beijing during the spring Asian dust season were shown in Table 1. Abundance of bacteria on dust particles was between $1 \times 10^{8}$ and $2 \times 10^{9}$ (average: $8 \times 10^{8}$ ) cells/g. The source region of Asian dust particles during this Asian dust season was determined to be the Gobi Desert by HYSPLIT back trajectory analysis (http://ready.arl.noaa.gov/HYSPLIT_traj.php). We have determined bacterial abundance in the Gobi Desert to be between $5 \times 10^{6}$ and $4 \times 10^{8}$ (average: $9 \times 10^{7}$ ) cells/g sand. ${ }^{35)}$ The number of bacterial cells transported to Beijing with Asian dust particles was about 10 times higher than those in the dust source regions. Bacterial abundance in general soil environments ranges from $10^{10}-10^{11}$ cells/g. ${ }^{36)}$ Soil particles which are lifted up from the ground by strong wind can be mixed with Asian dust particles during transportation ${ }^{37)}$ from the dust source region to Beijing, and thus bacterial abundance of Asian dust particles could increase.

Inter-Seasonal Variability of Bacterial Community Structure during Asian Dust Season T-RFLP targeting the bacterial 16S rRNA gene as a universal genetic marker can be widely used for characterization of bacterial community composition. ${ }^{29)}$ T-RFLP is semi-quantitative and has a high reproducibility. T-RFLP has been applied for community analysis of bacteria in natural environments (e.g., lakes, ${ }^{38)}$ ocean, ${ }^{39)}$ soil $^{40)}$ and air $^{24)}$ ), because this method is suitable for evaluation of temporal and spatial changes in the targeted microbial communities. T-RFLP analysis was therefore performed in this study with DNA fragments obtained from 12 Asian dust samples, to examine the similarity of bacterial communities ${ }^{41}$ ) 
among Asian dust samples. The T-RFLP profiles were similar among the 12 samples, and two dominant OTUs with lengths of 150 and $275 \mathrm{bp}$ were detected in all Asian dust samples (Fig. 3) while some specific OTUs (445 and $495 \mathrm{bp}$ ) were detected in a few samples collected on 29 April and 7 May. These measured peak profiles were analyzed by the database MiCA3. Bacterial species producing the $150 \mathrm{bp}$ fragment under the T-RFLP conditions used in this study were Azospirillum brasilense (Proteobacteria; nitrogen-fixing bacteria), Azospirillum zeae (Proteobacteria; nitrogen-fixing bacteria), Beijerinckia indica (Proteobacteria; nitrogen-fixing bacteria), Methylobacterium nodulans (Proteobacteria; nitrogen-fixing bacteria), Methylobacterium radiotolerans (Proteobacteria;

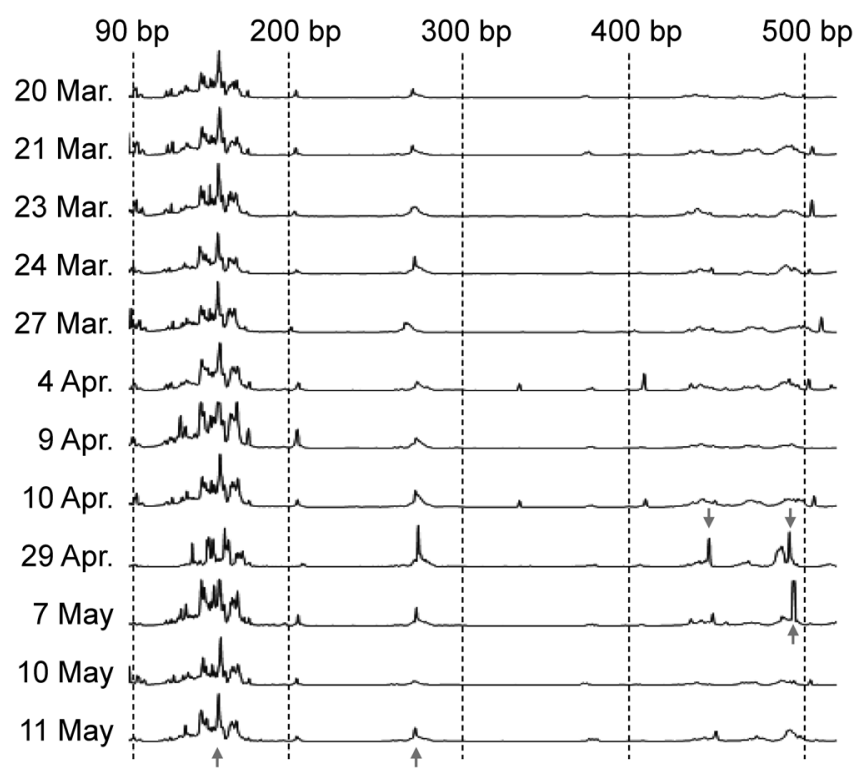

Fig. 3. T-RFLP Profiles of 16S rRNA Genes Obtained from 12 Asian Dust Samples Collected in Beijing

Arrows indicate 150, 275, 445 and $495 \mathrm{bp}$, respectively. radiation tolerating bacteria), Paenibacillus polymyxa (Firmicutes; nitrogen-fixing bacteria), Sphingobium japonicum (Proteobacteria; soil bacteria), Sphingomonas melonis (Proteobacteria; plant pathogenic bacteria). In addition, Bacillus sp. (spore-forming bacteria frequently isolated from soil), uncultured Bacteroidetes bacterium and Rubellimicrobium sp. (atmospheric bacteria) also produce the $150 \mathrm{bp}$ fragment under the T-RFLP conditions used in this study. Bacteria producing the 275, 445 and 495 bp length fragments were Corynebacterium pseudotuberculosis (Actinobacteria; frequently isolated from soil and pathogen of livestock ${ }^{42)}$ ), Polynucleobacter nec-

Table 2. Sequence Similarity to Closest Relatives and Phylogenetic Affiliation of Bacterial DNA Collected from Asian Dust Samples and Recovered from the DGGE Gel (Fig. 4A)

\begin{tabular}{cclc}
\hline \hline & Band & \multicolumn{1}{c}{ Closely related species } & $\begin{array}{c}\text { Number of matched } \\
\text { bases (similarity) }\end{array}$ \\
\hline 20 Mar. & A & Hymenobacter sp. & $444 / 456(97 \%)$ \\
& B & Paracoccus sp. & $401 / 431(93 \%)$ \\
& C & Methylobacteriaceae bacterium & $451 / 454(99 \%)$ \\
D & Shinella sp. & $442 / 457(96 \%)$ \\
E & Rubellimicrobium aerolatum & $416 / 435(95 \%)$ \\
F & Leptolyngbya sp. & $440 / 455(96 \%)$ \\
G & Planococcus sp. & $457 / 457(100 \%)$ \\
H & Rubellimicrobium sp. & $313 / 324(96 \%)$ \\
I & Rubellimicrobium sp. & $428 / 433(98 \%)$ \\
J & Skermanella sp. & $453 / 458(98 \%)$ \\
\hline 10 May & Adhaeribacter aquaticus & $440 / 459(95 \%)$ \\
L & Niastella koreensis & $438 / 457(95 \%)$ \\
M & Methylobacteriaceae bacterium & $457 / 459(99 \%)$ \\
N & Kaistia sp. & $438 / 459(95 \%)$ \\
O & Shinella sp. & $440 / 457(96 \%)$ \\
P & Hymenobacter sp. & $453 / 456(99 \%)$ \\
Q & Rubellimicrobium aerolatum & $412 / 430(95 \%)$ \\
R & Rubellimicrobium sp. & $428 / 433(98 \%)$ \\
S & Skermanella sp. & $453 / 458(98 \%)$ \\
\hline
\end{tabular}

(B)

(A)
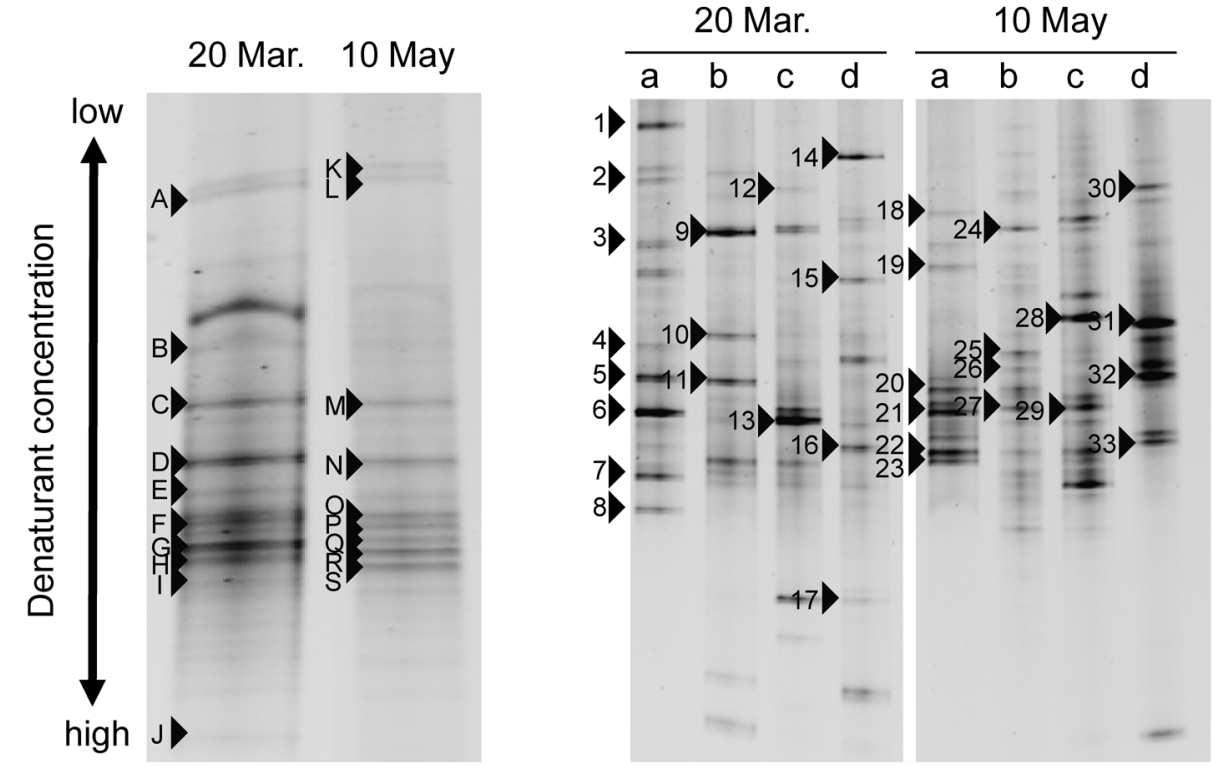

Fig. 4. DGGE Profiles of the 16S rRNA Gene Obtained from Asian Dust Samples Collected in Beijing during the Spring Dust Season (A) Uncultured dust samples. (B) Dust samples cultured with a) standard medium, b) 1\% standard medium, c) $0.05 \%$ yeast extract, and d) R2A medium. 
Table 3. Sequence Similarity to Closest Relatives and Phylogenetic Affiliation of Bacterial DNA Collected from Cultured Asian Dust Samples and Recovered from the DGGE Gel (Fig. 4B)

\begin{tabular}{|c|c|c|c|}
\hline & Band & Closely related species & $\begin{array}{c}\text { Number of matched } \\
\text { bases (similarity) }\end{array}$ \\
\hline \multirow[t]{17}{*}{20 Mar. } & 1 & Clostridium aciditolerans & $444 / 452(98 \%)$ \\
\hline & 2 & Clostridium botulinum & $425 / 445(95 \%)$ \\
\hline & 3 & Clostridium sp. & $450 / 452(99 \%)$ \\
\hline & 4 & Clostridium sp. & $433 / 452(95 \%)$ \\
\hline & 5 & Salmonella enterica & $451 / 456(98 \%)$ \\
\hline & 6 & Salmonella enterica & $453 / 456(99 \%)$ \\
\hline & 7 & Erwinia sp. & $450 / 456(98 \%)$ \\
\hline & 8 & Erwinia sp. & $455 / 456(99 \%)$ \\
\hline & 9 & Bifissio spartinae & $445 / 457(97 \%)$ \\
\hline & 10 & Adhaeribacter aquaticus & $452 / 456(99 \%)$ \\
\hline & 11 & Magnetospirillum bellicus & $455 / 458(99 \%)$ \\
\hline & 12 & Sphingobacterium sp. & $439 / 457(96 \%)$ \\
\hline & 13 & Telmatospirillum siberiense & $437 / 459(95 \%)$ \\
\hline & 14 & Bacteroidetes bacterium & $413 / 455(90 \%)$ \\
\hline & 15 & Bacteroidetes bacterium & $413 / 456(90 \%)$ \\
\hline & 16 & Methylophaga thalassica & $405 / 455(89 \%)$ \\
\hline & 17 & Dechlorospirillum sp. & $441 / 455(96 \%)$ \\
\hline \multirow[t]{16}{*}{10 May } & 18 & Clostridium algidixylanolyticum & $343 / 345(99 \%)$ \\
\hline & 19 & Clostridium sp. & $451 / 453(99 \%)$ \\
\hline & 20 & Clostridiaceae bacterium & $449 / 453(99 \%)$ \\
\hline & 21 & Ensifer $\mathrm{sp}$ & $459 / 459(100 \%)$ \\
\hline & 22 & Pseudomonas sp. & $457 / 457(100 \%)$ \\
\hline & 23 & Cupriavidus necator & $450 / 452(99 \%)$ \\
\hline & 24 & Flavosolibacter ginsengiter & $454 / 455(99 \%)$ \\
\hline & 25 & Flavosolibacter sp. & $447 / 457(97 \%)$ \\
\hline & 26 & Deinococcus sp. & $452 / 459(98 \%)$ \\
\hline & 27 & Microvirga sp. & $459 / 460(99 \%)$ \\
\hline & 28 & Pedobacter ginsengisoli & $453 / 456(99 \%)$ \\
\hline & 29 & Flavobacterium sp. & $445 / 454(98 \%)$ \\
\hline & 30 & Bacteroidetes bacterium & $449 / 456(98 \%)$ \\
\hline & 31 & Clostridium magnum & $440 / 453(97 \%)$ \\
\hline & 32 & Bacillus sp. & $455 / 455(100 \%)$ \\
\hline & 33 & Stenotrophomonas sp. & $456 / 458(99 \%)$ \\
\hline
\end{tabular}

essaries (Proteobacteria; free-living freshwater bacteria) and Salmonella enterica (Proteobacteria; intestinal bacteria), respectively. These results are summarized that nitrogen-fixing bacteria frequently found in natural environments, especially in soil, were detected and some pathogenic and intestinal bacteria were also found by T-RFLP analysis.

Viable Bacteria in Asian Dust Collected in Beijing DGGE was used to determine the viable bacterial community in Asian dust samples collected in Beijing. The sequences of 52 bands on DGGE gels were determined (19 bands from uncultured Asian dust samples (Fig. 4A) and 33 bands from cultured Asian dust samples (Fig. 4B). Phylogenetically diverse bacteria were confirmed to exist in the Asian dust samples (Tables 2, 3).

In uncultured bacterial population (Table 2), soil bacteria such as Hymenobacter sp., Paracoccus sp., Methylobacteriaceae, Shinella sp., Planococcus sp., Skermanella sp., Niastella koreensis, Kaistia sp. were found as well as atmospheric bacteria (Rubellimicrobium spp.) and aquatic bacteria (Adhaeribacter aquaticus). As shown in Table 2, Hymenobacter sp., Methylobacteriaceae, Shinella sp., Rubellimicrobium spp. and Skermanella sp. were found in the both Asian dust samples

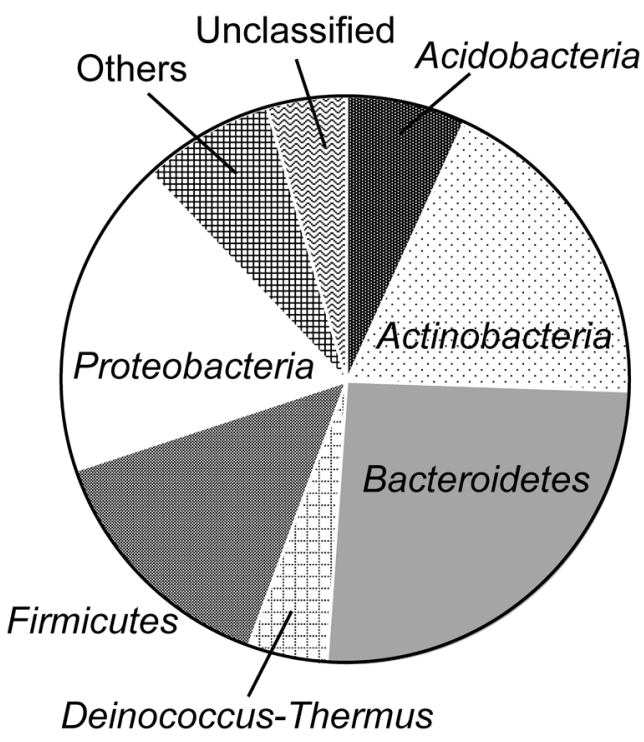

Fig. 5. Relative Abundances of the Most Common Bacterial Phyla in Asian Dust Collected in Beijing

collected in March and May.

In cultured Asian dust samples (Table 3), soil bacteria such as Clostridium spp., Ensifer sp., Flavosolibacter spp., Microvirga sp., Pedobacter ginsengisoli, Bacillus sp. and Stenotrophomonas sp. were detected. As shown in Table 3, Clostridium spp. and uncultured Bacteroidetes bacterium were found in the both Asian dust samples collected in March and May. In addition to these bacteria found in natural environments, intestinal bacteria (Salmonella enterica and Erwinia sp.) were detected. Salmonella enterica were also detected in the T-RFLP analysis as previously described. These intestinal bacteria are sometimes found in fecal compost, and they may be recent dust contaminants from soil environments with fecal compost between Beijing and the dust source area, because these intestinal bacteria are thought to lose their activities by UV exposure and desiccation during transportation. Magnetospirillum bellicus and Dechlorospirillum sp. were also detected and these bacteria were described in DDBJ as found in activated sludge used in wastewater treatment plants. There are several wastewater treatment plants around Beijing (http://www.gcus.jp/report/wholeReport/document/pdf/ gesuidouten2010_1-03.pdf) and these data may therefore support the explanation for the increase in bacterial abundance during transportation from the dust source area to Beijing, as previously mentioned in relation to results in Table 1.

Methylobacteriaceae, Rubellimicrobium sp., Salmonella enterica, uncultured Bacteroidetes bacterium, and Bacillus sp. were detected in the both T-RFLP and DGGE analysis.

Phylogenetic Analysis of Asian Dust Bacterial Community The bacterial community on Asian dust particles was further analyzed using a clone library targeting the bacterial 16S rRNA gene. As mentioned above, T-RFLP analysis indicated that bacterial communities were similar among 12 Asian dust samples, and phylogenetic analysis was therefore performed on the Asian dust sample collected on 20 March. As shown in Fig. 5, major phyla identified from the dust sample were the members of Bacteroidetes (26\%), Actinobacteria (19\%), Proteobacteria (18\%) and Firmicutes (14\%), and this result was similar to the results reported in previous 
Table 4. Principal Phylotypes of Clones Obtained from 16S rRNA Gene Clone Library

\begin{tabular}{|c|c|c|c|}
\hline Accession No. & Phylum & Family & Closest described species \\
\hline LC026921 & Acidobacteria & Acidobacteriaceae & Acidobacteria bacterium Ac_11_E3 \\
\hline LC026871 & Acidobacteria & Acidobacteriaceae & Acidobacteria bacterium KBS 96 \\
\hline LC026841 & Acidobacteria & Acidobacteriaceae & Acidobacteria bacterium LP6 \\
\hline LC026877 & Acidobacteria & Acidobacteriaceae & Acidobacteria bacterium LWQ4 \\
\hline LC026880 & Acidobacteria & Acidobacteriaceae & Acidobacteria bacterium LWQ4 \\
\hline LC026907 & Acidobacteria & Acidobacteriaceae & Acidobacteria bacterium WWH8 \\
\hline LC026844 & Acidobacteria & Blastocatellaceae & Aridibacter kavangonensis \\
\hline $\mathrm{LC} 026845$ & Acidobacteria & Blastocatellaceae & Aridibacter kavangonensis \\
\hline LC026933 & Acidobacteria & Blastocatellaceae & Blastocatella fastidiosa \\
\hline LC026932 & Actinobacteria & Pseudonocardiaceae & Actinomycetospora straminea \\
\hline LC026815 & Actinobacteria & Micrococcaceae & Arthrobacter globiformis \\
\hline LC026857 & Actinobacteria & Micrococcaceae & Arthrobacter phenanthrenivorans \\
\hline LC026899 & Actinobacteria & Micrococcaceae & Arthrobacter phenanthrenivorans \\
\hline LC026838 & Actinobacteria & Cellulomonadaceae & Cellulomonas aerilata \\
\hline LC026931 & Actinobacteria & Cellulomonadaceae & Cellulomonas aerilata \\
\hline LC026903 & Actinobacteria & Microbacteriaceae & Curtobacterium sp. BM-8J \\
\hline LC026840 & Actinobacteria & Nocardioidaceae & Friedmanniella lacustris \\
\hline LC026897 & Actinobacteria & Nocardioidaceae & Friedmanniella lacustris \\
\hline LC026924 & Actinobacteria & Nocardioidaceae & Friedmanniella lacustris \\
\hline LC026925 & Actinobacteria & Nocardioidaceae & Friedmanniella lacustris \\
\hline LC026856 & Actinobacteria & Nocardioidaceae & Friedmanniella luteola \\
\hline LC026864 & Actinobacteria & Nocardioidaceae & Friedmanniella luteola \\
\hline LC026891 & Actinobacteria & Nocardioidaceae & Friedmanniella luteola \\
\hline LC026818 & Actinobacteria & Nocardioidaceae & Friedmanniella sp. I10A-01996 \\
\hline LC026843 & Actinobacteria & Nocardioidaceae & Friedmanniella sp. I10A-01996 \\
\hline LC026866 & Actinobacteria & Nocardioidaceae & Friedmanniella spumicola \\
\hline LC026833 & Actinobacteria & Nocardioidaceae & Nocardioides ganghwensis \\
\hline LC026826 & Actinobacteria & & Nostocoida limicola \\
\hline LC026859 & Actinobacteria & & Nostocoida limicola \\
\hline LC026900 & Actinobacteria & & Nostocoida limicola \\
\hline LC026893 & Actinobacteria & Micromonosporaceae & Phytohabitans rumicis \\
\hline LC026869 & Actinobacteria & Pseudonocardiaceae & Pseudonocardia alaniniphila \\
\hline LC026878 & Actinobacteria & Pseudonocardiaceae & Pseudonocardia alaniniphila \\
\hline LC026892 & Actinobacteria & Rubrobacteraceae & Rubrobacter xylanophilus \\
\hline LC026853 & Armatimonadetes & Armatimonadaceae & Armatimonas rosea \\
\hline LC026917 & Armatimonadetes & Armatimonadaceae & Armatimonas rosea \\
\hline LC026852 & Bacteroidetes & Cytophagaceae & Adhaeribacter sp. TSX13-1 \\
\hline LC026911 & Bacteroidetes & & Bacteroidetes bacterium RG1-1 \\
\hline LC026922 & Bacteroidetes & Chitinophagaceae & Chitinophaga niabensis \\
\hline LC026901 & Bacteroidetes & Cytophagaceae & Cytophagaceae bacterium MCCP1 \\
\hline LC026823 & Bacteroidetes & Flammeovirgaceae & Flammeovirgaceae bacterium 311 \\
\hline LC026851 & Bacteroidetes & Chitinophagaceae & Flavisolibacter ginsengisoli \\
\hline LC026822 & Bacteroidetes & Chitinophagaceae & Flavisolibacter ginsengiterrae \\
\hline LC026916 & Bacteroidetes & Chitinophagaceae & Flavisolibacter sp. MDT2-37 \\
\hline LC026819 & Bacteroidetes & Cytophagaceae & Hymenobacter fastidiosus \\
\hline LC026915 & Bacteroidetes & Cytophagaceae & Hymenobacter fastidiosus \\
\hline LC026904 & Bacteroidetes & Cytophagaceae & Hymenobacter qilianensis \\
\hline LC026940 & Bacteroidetes & Cytophagaceae & Hymenobacter sp. DG31A \\
\hline LC026936 & Bacteroidetes & Cytophagaceae & Hymenobacter sp. R-37565 \\
\hline LC026941 & Bacteroidetes & Cytophagaceae & Hymenobacter sp. R-37565 \\
\hline LC026908 & Bacteroidetes & Cytophagaceae & Hymenobacter sp. VUG-A141a \\
\hline LC026926 & Bacteroidetes & Cytophagaceae & Hymenobacter sp. VUG-A60a \\
\hline LC026860 & Bacteroidetes & Cytophagaceae & Pontibacter diazotrophicus \\
\hline LC026930 & Bacteroidetes & Cytophagaceae & Pontibacter korlensis \\
\hline LC026842 & Bacteroidetes & Cytophagaceae & Pontibacter sp. MDT1-10-3 \\
\hline LC026867 & Bacteroidetes & Cytophagaceae & Rhodocytophaga aerolata \\
\hline LC026875 & Bacteroidetes & Cytophagaceae & Rhodocytophaga aerolata \\
\hline $\mathrm{LC} 026863$ & Bacteroidetes & Cytophagaceae & Rufibacter tibetensis \\
\hline LC026817 & Bacteroidetes & Chitinophagaceae & Segetibacter aerophilus \\
\hline LC026820 & Bacteroidetes & Chitinophagaceae & Segetibacter aerophilus \\
\hline
\end{tabular}


Table 4. Continued

\begin{tabular}{|c|c|c|c|}
\hline Accession No. & Phylum & Family & Closest described species \\
\hline LC026831 & Bacteroidetes & Chitinophagaceae & Segetibacter aerophilus \\
\hline LC026836 & Bacteroidetes & Chitinophagaceae & Segetibacter aerophilus \\
\hline LC026839 & Bacteroidetes & Chitinophagaceae & Segetibacter aerophilus \\
\hline LC026848 & Bacteroidetes & Chitinophagaceae & Segetibacter aerophilus \\
\hline LC026870 & Bacteroidetes & Chitinophagaceae & Segetibacter aerophilus \\
\hline LC026879 & Bacteroidetes & Chitinophagaceae & Segetibacter aerophilus \\
\hline LC026887 & Bacteroidetes & Chitinophagaceae & Segetibacter aerophilus \\
\hline LC026906 & Bacteroidetes & Chitinophagaceae & Segetibacter aerophilus \\
\hline LC026868 & Bacteroidetes & Cytophagaceae & Sporocytophaga myxococcoides \\
\hline LC026876 & Bacteroidetes & Cytophagaceae & Sporocytophaga myxococcoides \\
\hline LC026938 & Chloroflexi & & Green non-sulfur bacterium AK-6 \\
\hline LC026942 & Chloroflexi & Oscillochloridaceae & Oscillochloris trichoides DG-6 \\
\hline LC026902 & Cyanobacteria & & Gloeocapsopsis crepidinum LEGE 06123 \\
\hline LC026835 & Cyanobacteria & & Trichocoleus sociatus SAG 26.92 \\
\hline LC026847 & Deinococcus-Thermus & Deinococcaceae & Deinococcus deserti VCD115 \\
\hline LC026872 & Deinococcus-Thermus & Deinococcaceae & Deinococcus sp. 4B6 \\
\hline LC026881 & Deinococcus-Thermus & Deinococcaceae & Deinococcus sp. 4B6 \\
\hline LC026837 & Deinococcus-Thermus & Trueperaceae & Truepera radiovictrix DSM 17093 \\
\hline LC026889 & Deinococcus-Thermus & Trueperaceae & Truepera radiovictrix DSM 17093 \\
\hline LC026913 & Deinococcus-Thermus & Trueperaceae & Truepera radiovictrix DSM 17093 \\
\hline LC026914 & Deinococcus-Thermus & Trueperaceae & Truepera radiovictrix DSM 17093 \\
\hline LC026811 & Firmicutes & Bacillaceae & Bacillus sp. IDA3504 \\
\hline LC026855 & Firmicutes & Bacillaceae & Bacillus sp. IHB B 2252 \\
\hline LC026827 & Firmicutes & Bacillaceae & Bacillus sp. ISO_06_Kulunda \\
\hline LC026894 & Firmicutes & Bacillaceae & Bacillus sp. LCP70 \\
\hline LC026886 & Firmicutes & Carnobacteriaceae & Carnobacterium sp. ARCTIC-P35 \\
\hline LC026849 & Firmicutes & Paenibacillaceae & Paenibacillus sp. 6M01 \\
\hline LC026937 & Firmicutes & Planococcaceae & Planococcus antarcticus \\
\hline LC026939 & Firmicutes & Planococcaceae & Planococcus maritimus \\
\hline LC026912 & Firmicutes & Planococcaceae & Planococcus sp. B17 \\
\hline LC026829 & Firmicutes & Planococcaceae & Planococcus sp. PAMC 21323 \\
\hline LC026832 & Firmicutes & Planococcaceae & Planococcus sp. PAMC 21323 \\
\hline LC026821 & Firmicutes & Planococcaceae & Planococcus sp. YIM C738 \\
\hline LC026910 & Firmicutes & Planococcaceae & Planococcus sp. YIM C738 \\
\hline LC026920 & Firmicutes & Planococcaceae & Planomicrobium glaciei \\
\hline LC026828 & Firmicutes & Planococcaceae & Planomicrobium koreense \\
\hline LC026888 & Firmicutes & Planococcaceae & Planomicrobium sp. XN13 \\
\hline LC026890 & Firmicutes & Planococcaceae & Planomicrobium sp. XN13 \\
\hline LC026812 & Firmicutes & Veillonellaceae & Sporomusa paucivorans \\
\hline LC026884 & Firmicutes & Thermoactinomycetaceae & Thermoactinomyces sp. T36 \\
\hline LC026885 & Nitrospirae & Nitrospiraceae & Nitrospira sp. \\
\hline LC026943 & Planctomycetes & Planctomycetaceae & Planctomycetaceae bacterium LX124 \\
\hline LC026935 & Planctomycetes & Planctomycetaceae & Planctomycetaceae bacterium WSF3-27 \\
\hline LC026830 & Proteobacteria & Rhodobacteraceae & Agaricicola taiwanensis \\
\hline LC026862 & Proteobacteria & Alteromonadaceae & Alteromonas sp. Gp-4-13.1 \\
\hline LC026898 & Actinobacteria & Micrococcaceae & Arthrobacter phenanthrenivorans \\
\hline LC026834 & Proteobacteria & Rhodospirillaceae & Azospirillum sp. LH-CAB12 \\
\hline LC026850 & Proteobacteria & Rhodospirillaceae & Azospirillum sp. LH-CAB12 \\
\hline LC026905 & Proteobacteria & & Bacterium Ellin5074 \\
\hline LC026816 & Proteobacteria & & Candidatus Entotheonella palauensis \\
\hline LC026874 & Proteobacteria & Acetobacteraceae & Craurococcus roseus \\
\hline LC026883 & Proteobacteria & Acetobacteraceae & Craurococcus roseus \\
\hline LC026928 & Proteobacteria & & Delta proteobacterium LX33 \\
\hline LC026825 & Proteobacteria & Oxalobacteraceae & Massilia sp. B48 \\
\hline LC026896 & Proteobacteria & Phyllobacteriaceae & Nitratireductor $\mathrm{sp} . \mathrm{ZZ}-1$ \\
\hline LC026919 & Proteobacteria & Nitrosomonadaceae & Nitrosomonas sp. Is79A3 \\
\hline LC026909 & Proteobacteria & Oxalobacteraceae & Oxalobacter sp. W1.09-142 \\
\hline LC026927 & Proteobacteria & Polyangiaceae & Polyangium fumosum \\
\hline LC026814 & Proteobacteria & Rhodobacteraceae & Rubellimicrobium mesophilum DSM 19309 \\
\hline LC026861 & Proteobacteria & Rhodobacteraceae & Rubellimicrobium roseum \\
\hline
\end{tabular}


Table 4. Continued

\begin{tabular}{|c|c|c|c|}
\hline Accession No. & Phylum & Family & Closest described species \\
\hline LC026813 & Proteobacteria & Rhodospirillaceae & Skermanella sp. Py-2-1 \\
\hline LC026895 & Proteobacteria & Rhodospirillaceae & Skermanella sp. Py-2-1 \\
\hline LC026923 & Proteobacteria & Rhodospirillaceae & Skermanella sp. Py-2-1 \\
\hline LC026929 & Proteobacteria & Sorangiineae & Sorangiineae bacterium SBSr006 \\
\hline LC026873 & Proteobacteria & Sphingomonadaceae & Sphingomonas sediminicola \\
\hline LC026882 & Proteobacteria & Sphingomonadaceae & Sphingomonas sediminicola \\
\hline LC026865 & Unclassified & & Bacterium Ellin6505 \\
\hline LC026934 & Unclassified & & Bacterium LWQ8 \\
\hline LC026918 & Unclassified & & Bacterium LY17 \\
\hline LC026846 & Unclassified & & Bacterium WHC1-2 \\
\hline LC026854 & Unclassified & & Bacterium YC-LK-LKJ4 \\
\hline LC026858 & Unclassified & & Unidentified eubacterium clone BSV87 \\
\hline LC026824 & Verrucomicrobia & & Verrucomicrobia bacterium WY51 \\
\hline
\end{tabular}

studies on phylogenetic analysis of bacterial composition in aeolian dust. ${ }^{1,2}$ Jeon et al. collected Asian dust samples in Korea and reported that dominant bacterial phyla were the members of Firmicutes, Actinobacteria and Proteobacteria. ${ }^{43)}$ The obtained clone library also supported the results obtained by T-RFLP analysis with the MiCA3 database and DGGE analysis. Actually, uncultured Bacteroidetes bacteria, Rubellimicrobium spp. (Proteobacteria; soil bacteria) and Bacillus spp. (Firmicutes; spore-forming bacteria frequently isolated from soil) were detected in the all analyses (T-RFLP, DGGE and clone library). In the clone library (Table 4), one clone of Bacteroidetes (unclassified to species level by DDBJ), two clones of Rubellimicrobium (one was R. mesophilum ${ }^{44)}$ and the other was $R$. roseum ${ }^{45)}$ ) and four clones of Bacillus spp. (all were unclassified to species level) were obtained.

In addition, we obtained several clones which were detected in the both DGGE analysis and clone library but not in the T-RFLP analysis; three clones of Deinococcus sp. (atmospheric bacteria; one was $D$. deserti and the others were unclassified to species level), as well as clones of soil bacteria such as three clones of Skermanella sp. (all unclassified to species level), seven clones of Planococcus sp. (one was $P$. antarcticus, one was $P$. maritimus and five were unclassified to species level) and eight clones of Hymenobacter sp. (two clones were $H$. fastidiosus, one clone was $H$. qilianensis and five clones were unclassified to species level). Also, as shown in Table 4, Segetibacter aerophilus (Bacteroidetes), which was first isolated from Asian dust samples collected in Korea with R2A agar, ${ }^{46)}$ was dominant and occupied $7.5 \%$ of the clone library.

As previously mentioned, the source region of Asian dust particles during this Asian dust season was determined to be the Gobi Desert by HYSPLIT back trajectory analysis. We have analyzed the bacterial community structure of desert soil of the Gobi Desert ${ }^{26)}$ and found it to be composed of Proteobacteria (36\%), Actinobacteria (24\%), Bacteroidetes (19\%) and Firmicutes $(6 \%)$. The bacterial community structure of Asian dust particles collected in Beijing was rather different from that of the dust source, the Gobi Desert. We have also analyzed bacterial community composition of Asian dust samples collected in Japan (Tottori Prefecture), 3000-5000 km from the dust source region ${ }^{26)}$ in spring. These Asian dust particles were collected at $900 \mathrm{~m}$ altitude by a sampler set in an airplane. The major phyla identified in this Asian dust sample were Firmicutes (30\%), Bacteroidetes (24\%) and Actinobacteria (23\%), and Proteobacteria (12\%). These data demonstrate that the ratio of Firmicutes gradually increased from dust source region (6\%) to Beijing (14\%) and Tottori (30\%), while Proteobacteria gradually decreased from dust source region (36\%) to Beijing (18\%) and to Tottori $(12 \%)$.

Smith et al. analyzed the community structure of airborne bacteria transported to North America (Mt. Bachelor Observatory, Oregon; altitude of $2800 \mathrm{~m}$ ) by Asian dust events, ${ }^{47)}$ and they reported that the dominant bacterial phyla in their aerosol samples were Firmicutes and Actinobacteria. The distances are approximately $1500 \mathrm{~km}$ from Beijing to Tottori and $7500 \mathrm{~km}$ from Beijing to Mt. Bachelor Observatory. Most bacteria transported by aeolian dust will be stressed by the conditions encountered during atmospheric transport (UV exposure, reduced nutrient availability and desiccation). Firmicutes are known for desiccation-tolerant and thus the percentages of this phylum was probably increased during long-range transportation of Asian dust particles.

\section{CONCLUSION}

In this study, we visualized and quantified bacterial cells on Asian dust particles collected in Beijing where heavy Asian dust particles fall at approximately $180 \mathrm{t} / \mathrm{km}^{2} /$ year and the influence of these dusts on health and ecosystems is of particular concern. Bacterial numbers on Asian dust particles seemed to increase compared with those in dust source regions. Interseasonal variability of bacterial community structure in Asian dust samples was low and it was assumed that bacterial community structure on dust particles changed during transportation. Results obtained in this study indicated that Asian dust contains high numbers of bacterial cells and their potential influence on health and ecosystems was demonstrated because some of those detected bacteria, including intestinal bacteria, remained viable.

We collected Asian dust samples at the top of a building in Beijing and therefore local soil particles might be mixed in the collected samples. When one has to analyze only the transported aeolian dust particles, one should collect dust particles at high altitude using balloons, helicopters or airplanes to avoid contamination of soil particles from the ground. ${ }^{26)} \mathrm{On}$ the other hand, one can collect dust particles near the ground when the purpose of the study is evaluation of the influence 
of those particles on our health and our surrounding living environment, because not only the transported particles but soil particles lifted up by wind near the sampling site can have the influence. Microbes in aeolian dust should have a greater influence in downwind areas near the dust source. Continuous temporal and spatial analyses from dust source regions to downwind regions will help to estimate the impact of atmospherically transported bacteria on health and ecosystems in downwind areas.

Acknowledgments This study was supported by the JSPS KAKENHI (25281030) and the Environment Research and Technology Development Fund of the Ministry of the Environment, Japan (B-0902).

Conflict of Interest The authors declare no conflict of interest.

\section{REFERENCES}

1) Griffin DW, Garrison VH, Herman JR, Shinn EA. African desert dust in the Caribbean atmosphere: microbiology and public health. Aerobiologia, 17, 203-213 (2001).

2) Griffin DW, Kellogg CA, Garrison VH, Lisle JT, Borden TC, Shinn EA. Atmospheric microbiology in the northern Caribbean during African dust events. Aerobiologia, 19, 143-157 (2003).

3) Prospero JM, Blades E, Mathison G, Naidu R. Interhemispheric transport of viable fungi and bacteria from Africa to the Caribbean with soil dust. Aerobiologia, 21, 1-19 (2005).

4) Griffin DW. Atmospheric movement of microorganisms in clouds of desert dust and implications for human health. Clin. Microbiol. Rev., 20, 459-477 (2007).

5) Hervàs A, Camarero L, Reche I, Casamayor EO. Viability and potential for immigration of airborne bacteria from Africa that reach high mountain lakes in Europe. Environ. Microbiol., 11, 1612-1623 (2009).

6) Iwasaka Y, Yamato M, Imasu R, Ono A. The transport of Asia dust (KOSA) particles; importance of weak KOSA events on the geochemical cycle of soil particles. Tellus, 40B, 494-503 (1988).

7) Duce RA, Unni CK, Ray BJ, Prospero JM, Merrill JT. Long-range atmospheric transport of soil dust from Asia to the tropical North Pacific: temporal variability. Science, 209, 1522-1524 (1980).

8) Kellogg CA, Griffin DW. Aerobiology and the global transport of desert dust. Trends Ecol. Evol., 21, 638-644 (2006).

9) Uno I, Eguchi K, Yumimoto K, Takemura T, Shimizu A, Uematsu M, Liu Z, Wang Z, Hara Y, Sugimoto N. Asian dust transported one full circuit around the globe. Nat. Geosci., 2, 557-560 (2009).

10) Bory AJ-M, Biscaye PE, Grousset FE. Two distinct seasonal Asian source regions for mineral dust deposited in Greenland (NorthGRIP). Geophys. Res. Lett., 30, 1167 (2003).

11) Grousset FE, Ginoux P, Bory A, Biscaye PE. Case study of a Chinese dust plume reaching the French Alps. Geophys. Res. Lett., 30, 1277 (2003).

12) Air Environment Division, Ministry of the Environment, Japan. "The Special Committee Report on Dust and Sandstorm Issues." 〈https://www.env.go.jp/air/dss/report/02/02_5.pdf〉, cited 10 October, 2015.

13) Nishikawa M, Mori I, Di Y, Quan H. Source impacts of fallout dust in Beijing. Proc. Internat. Aerosol Conference Taiwan, 433-434 (2002).

14) Yamaguchi N, Sakotani A, Ichijo T, Kenzaka T, Tani K, Baba T, Nasu M. Break down of Asian dust particle on wet surface and their possibilities of cause of respiratory health effects. Biol. Pharm. Bull., 35, 1187-1190 (2012).
15) Higashi T, Kambayashi Y, Ohkura N, Fujimura M, Nakanishi S, Yoshizaki T, Saijoh K, Hayakawa K, Kobayashi F, Michigami Y, Hitomi Y, Nakamura H. Exacerbation of daily cough and allergic symptoms in adult patients with chronic cough by Asian dust: A hospital-based study in Kanazawa. Atmos. Environ., 97, 537-543 (2014).

16) Honda A, Matsuda Y, Murayama R, Tsuji K, Nishikawa M, Koike E, Yoshida S, Ichinose T, Takano H. Effects of Asian sand dust particles on the respiratory and immune system. J. Appl. Toxicol., 34, 250-257 (2014).

17) Ren Y, Ichinose $T$, He M, Arashidani K, Yoshida Y, Yoshida S, Nishikawa M, Takano H, Sun G, Shibamoto T. Aggravation of ovalbumin-induced murine asthma by co-exposure to desert-dust and organic chemicals: an animal model study. Environ. Health, 13, 83 (2014).

18) Watanabe T, Hasei T, Kokunai O, Coulibaly S, Nishimura S, Fukasawa M, Takahashi R, Mori Y, Fujita K, Yoshihara Y, Miyake Y, Kishi A, Matsui M, Ikemori F, Funasaka K, Toriba A, Hayakawa K, Arashidani K, Inaba Y, Sera N, Deguchi Y, Seiyama T, Yamaguchi T, Watanabe M, Honda N, Wakabayashi K, Totsuka Y. Air pollution with particulate matter and mutagens: relevance of Asian dust to mutagenicity of airborne particles in Japan. Genes Environ., 36, $120-136$ (2014).

19) Hua N-P, Kobayashi F, Iwasaka Y, Shi G-Y, Naganuma T. Detailed identification of desert-originated bacteria carried by Asian dust storms to Japan. Aerobiologia, 23, 291-298 (2007).

20) Lee S, Choi B, Yi S, Ko G. Characterization of microbial community during Asian dust events in Korea. Sci. Total Environ., 407, 5308-5314 (2009).

21) Maki T, Susuki S, Kobayashi F, Kakikawa M, Tobo Y, Yamada M, Higashi T, Matsuki A, Hong C, Hasegawa H, Iwasaka Y. Phylogenetic analysis of atmospheric halotolerant bacterial communities at high altitude in an Asian dust (KOSA) arrival region, Suzu City. Sci. Total Environ., 408, 4556-4562 (2010).

22) Jeon E-M, Kim H-J, Jung K, Kim J-H, Kim M-Y, Kim YP, Ka J-O. Impact of Asian dust events on airborne bacterial community assessed by molecular analyses. Atmos. Environ., 45, 4313-4321 (2011).

23) Yamaguchi N, Nasu M. Flow cytometric analysis of bacterial respiratory and enzymatic activity in the natural aquatic environment. $J$. Appl. Microbiol., 83, 43-52 (1997).

24) Lee SH, Lee HJ, Kim SJ, Lee HM, Kang H, Kim YP. Identification of airborne bacterial and fungal community structures in an urban area by T-RFLP analysis and quantitative real-time PCR. Sci. Total Environ., 408, 1349-1357 (2010).

25) Maki T, Puspitasari F, Hara K, Yamada M, Kobayashi F, Hasegawa $\mathrm{H}$, Iwasaka Y. Variations in the structure of airborne bacterial communities in a downwind area during an Asian dust (Kosa) event. Sci. Total Environ., 488-489, 75-84 (2014).

26) Yamaguchi N, Ichijo T, Sakotani A, Baba T, Nasu M. Global dispersion of bacterial cells on Asian dust. Sci. Rep., 2, 525 (2012).

27) Iwamoto T, Tani K, Nakamura K, Suzuki Y, Kitagawa M, Eguchi M, Nasu M. Monitoring impact of in situ biostimulation treatment on groundwater bacterial community by DGGE. FEMS Microbiol. Ecol., 32, 129-141 (2000).

28) Nishimura Y, Kenzaka T, Sueyoshi A, Li P-F, Fujiyama H, Baba T, Yamaguchi N, Nasu M. Similarity of bacterial community structure between Asian dust and its sources determined by rRNA genetargeted approaches. Microbes Environ., 25, 22-27 (2010).

29) Liu W-T, Marsh TL, Cheng H, Forney LJ. Characterization of microbial diversity by determining terminal restriction fragment length polymorphisms of genes encoding $16 \mathrm{~S}$ rRNA. Appl. Environ. Microbiol., 63, 4516-4522 (1997).

30) Yamaguchi N, Matsukawa S, Shintome Y, Ichijo T, Nasu M. Microchip-based terminal restriction fragment length polymorphism for on-site analysis of bacterial communities in freshwater. Biol. 
Pharm. Bull., 36, 1305-1309 (2013).

31) Hodges TW, Olson JB. Molecular comparison of bacterial communities within iron-containing flocculent mats associated with submarine volcanoes along the Kermadec Arc. Appl. Environ. Microbiol., 75, 1650-1657 (2009).

32) Yamaguchi N, Park J, Kodama M, Ichijo T, Baba T, Nasu M. Change in the airborne bacterial community in outdoor environments following Asian dust events. Microbes Environ., 29, 82-88 (2014).

33) Cole JR, Wang Q, Cardenas E, Fish J, Chai B, Farris RJ, KulamSyed-Mohideen AS, McGarrell DM, Marsh T, Garrity GM, Tiedje JM. The Ribosomal Database Project: improved alignments and new tools for rRNA analysis. Nucleic Acids Res., 37 (Database), D141-D145 (2009).

34) Schloss PD, Handelsman J. Introducing DOTUR, a computer program for defining operational taxonomic units and estimating species richness. Appl. Environ. Microbiol., 71, 1501-1506 (2005).

35) Baba T, Ichijo T, Yamaguchi N, Nasu M. Diversity of bacteria transported by Asian dust events: bacterial community analysis based on rRNA genes. 7th Symposium on Atmospheric Bioaerosol, Shiga Prefectural University, Hikone, Japan (2013).

36) van der Heijden MGA, Bardgett RD, van Straalen NM. The unseen majority: soil microbes as drivers of plant diversity and productivity in terrestrial ecosystems. Ecol. Lett., 11, 296-310 (2008).

37) Tanaka T, Maki T, Ishimizu T, Ebita A, Chiba M. Development of a global mineral dust model and its application to the forecast of Kosa phenomena. J. Aerosol Res, 20, 297-305 (2005).

38) Schwarz JI, Eckert W, Conrad R. Community structure of Archaea and Bacteria in a profundal lake sediment Lake Kinneret (Israel). Syst. Appl. Microbiol., 30, 239-254 (2007).

39) Zhang R, Thiyagarajan V, Qian P-Y. Evaluation of terminal-restriction fragment length polymorphism analysis in contrasting marine environments. FEMS Microbiol. Ecol., 65, 169-178 (2008).

40) Singh BK, Nazaries L, Munro S, Anderson IC, Campbell CD. Use of multiplex terminal restriction fragment length polymorphism for rapid and simultaneous analysis of different components of the soil microbial community. Appl. Environ. Microbiol., 72, 7278-7285 (2006).

41) Zhang R, Thiyagarajan V, Qian P-Y. Evaluation of terminal-restriction fragment length polymorphism analysis in contrasting marine environments. FEMS Microbiol. Ecol., 65, 169-178 (2008).

42) Spier SJ, Toth B, Edman J, Quave A, Habasha F, Garrick M, Byrne BA. Survival of Corynebacterium pseudotuberculosis biovar equi in soil. Vet. Rec., 170, 180 (2012).

43) Jeon E-M, Kim H-J, Jung K, Kim J-H, Kim M-Y, Kim Y-P, Ka J-O. Impact of Asian dust events on airborne bacterial community assessed by molecular analyses. Atmos. Environ., 45, 4313-4321 (2011).

44) Dastager SG, Lee J-C, Ju Y-J, Park D-J, Kim C-J. Rubellimicrobium mesophilum sp. nov., a mesophilic, pigmented bacterium isolated from soil. Int. J. Syst. Evol. Microbiol., 58, 1797-1800 (2008).

45) Cao Y-R, Jiang Y, Wang Q, Tang S-K, He W-X, Xue Q-H, Xu L-H, Jiang C-L. Rubellimicrobium roseum sp. nov., a Gram-negative bacterium isolated from the forest soil sample. Antonie Van Leeuwenhoek, 98, 389-394 (2010).

46) Weon H-Y, Kwon S-W, Son J-A, Kim S-J, Kim Y-S, Kim B-Y, Ka J-O. Adhaeribacter aerophilus sp. nov., Adhaeribacter aerolatus sp. nov. and Segetibacter aerophilus sp. nov., isolated from air samples. Int. J. Syst. Evol. Microbiol., 60, 2424-2429 (2010).

47) Smith DJ, Timonen HJ, Jaffe DA, Griffin DW, Birmele MN, Perry $\mathrm{KD}$, Ward PD, Roberts MS. Intercontinental dispersal of bacteria and archaea by transpacific Winds. Appl. Environ. Microbiol., 79, 1134-1139 (2013). 